\title{
Dimensionality of childhood psychopathology and the challenge of integration into clinical practice
}

\author{
Guilherme V. Polanczyk
}

Published online: 29 March 2014

(C) Springer-Verlag Berlin Heidelberg 2014

For decades, the debate on whether psychopathology is best conceptualized as a dimension or as a category has occupied a central role in our field. Several articles and reviews were published on this theme [1-3]. In the past years, a growing body of evidence has suggested that dimensionality would be a more 'natural' approach to mental disorders, at least for some of them $[3,4]$. Thus, when the DSM-5 was initially planned, a dimensional component was supposed to be incorporated into the new classification system, in a way that patients could benefit from the understanding that the complexity of psychopathology is not entirely captured by diagnostic categories [5]. Unfortunately, this task was too ambitious for the moment and was not fully achieved, resulting in a final document that is fundamentally unchanged from the previous version. The DSM-5, as DSM-IV, is founded on categories and clinical decisions are still based on answers to dichotomous questions, with little impact of a dimensional component.

G. V. Polanczyk ( $\square)$

Department of Psychiatry, University of São Paulo Medical

School, São Paulo, Brazil

e-mail: gvp@usp.br

G. V. Polanczyk

National Institute of Developmental Psychiatry for Children and Adolescents (INCT-CNPq), São Paulo, Brazil

G. V. Polanczyk

Research Support Center on Neurodevelopment and Mental Health, University of São Paulo, São Paulo, Brazil

G. V. Polanczyk

Rua Dr. Ovídio Pires de Campos 785, Cerqueira César,

São Paulo, SP 05403-010, Brazil
In appreciation of the challenge task that is to incorporate dimensionality of psychopathology into the clinical world, where patients and families claim for definite answers about diagnosis and physicians routinely face difficult decisions about treatments, and also considering that we still have fundamental questions to be answered about the phenomenon we deal with, the National Institute of Mental Health launched the Research Domain Criteria (RDoC) project [6]. The project aims to create a framework for research on pathophysiology by not constraining the studies to diagnostic categories, but to clinical features. The primary focus for RDoC is on neural circuitry, with genetic variations, molecular and cellular factors, circuitry function, cognition and clinical manifestations as different levels of analysis. The aim of the project is to generate new knowledge that eventually may support significant advances in the clinical world, such as the identification of new targets for treatment development and the detection of subgroups for treatment selection [6]. To reach these goals, important modifications in the way research projects in our field are conceptualized and implemented will be necessary and considerable efforts in this direction are already in place.

The current issue of the European Child and Adolescent Psychiatry (ECAP) bring good examples of studies that conceptualize psychopathology as dimensional construct, aggregate different level of analysis, and investigate its manifestations beyond diagnostic categories. The study by Robel et al. [7] investigated the presence of autistic traits in siblings and parents of children with autistic spectrum disorder (ASD), using the self-report French Autism Quotient. Scores were distributed between two main factors, (1) socialization and communication, and (2) imagination and rigidity. Parents and siblings of probands presented higher scores than controls in the domains of 
communication and socialization. Additionally, using a latent approach to analyze the data, authors identified subgroups of parents of children with ASD according to scores on each of the two factors. Based on these results, authors discuss that each clinically defined subgroup may correspond to different underlying genetic mechanisms. The study by Nilsson et al. [8] examined a functionally relevant variation of the transcription factor activating protein-2 $\beta$ gene, which has been shown to influence monoaminergic neurotransmission, in the context of cooccurring symptoms of ADHD and depression. The authors investigated two independent population-based samples of adolescents, and replicated the finding that the presence of the $9 \mathrm{R}$ variant of the polymorphism, which is associated with increased gene expression, protects females with symptoms of ADHD from developing symptoms of depression.

Fuhrmann et al. [9] and Theunissen et al. [10] also study psychopathology as a dimensional construct with a focus on largely understudied populations. Fuhrmann et al. investigate the prevalence of depressive symptoms and co-occurring risk factors in a population-based sample of preschool children. The prevalence of depressive symptoms of clinical relevance was $5.7 \%$ with no difference between sexes. Depressive symptoms were associated with developmental motor and speech problems and disorders. Theunissen et al. [10] investigated behavioral problems in hearing-impaired children with hearing aids or cochlear implants, compared to normally hearing children. Hearing-impaired children showed more proactive aggression, more symptoms of conduct disorder, psychopathy, opposition, inattention, and hyperactivity. As the study by Theunissen et al. [10], the case report by Mazzei et al. [11] addresses the relationship of emotional and behavioral symptoms and other medical conditions, specifically homocystinuria-an inborn error of metabolism-as a cause of impairment of early neurodevelopment and several psychopathological manifestations, including psychosis.

Finally, the study by Posserud et al. [12] touched on a related and very sensitive issue: on the agreement between screening and diagnostic instruments. Authors showed a fair to moderate agreement for ADHD. This is a very important aspect, since rating scales and diagnostic instruments are widely used to measure behavior, and may be doing so in very different ways, which can only result in divergent findings. This study indicates the need of developing more objective measures for behavior. Lack of strong measures also affects the possibility of measuring outcomes in routine psychiatric care, as shown by the review of Hall et al. [13], thus limiting the assessment and improvement of services and resulting in significant backlogs to our field.
The studies in this issue of the ECAP are excellent examples of proficuous investigations that are looking beyond categorical diagnostic criteria. As we would expect, there are limitations for this approach, such as the psychometric properties of instruments used, and how results can be translated to clinical practice [14]. Significant advances need to be made to overcome methodological limitations, and these are urgent. The question of how the knowledge that is being produced will be integrated into the clinical world will depend on our scientific rigor, creativity, and innovative thinking in conducting the next generation of studies.

\section{References}

1. Pagsberg AK (2013) Schizophrenia spectrum and other psychotic disorders. Eur Child Adolesc Psychiatry 22(Suppl 1):S3-S9

2. Rutter M (2011) Research review: child psychiatric diagnosis and classification: concepts, findings, challenges and potential. J Child Psychol Psychiatry 52(6):647-660

3. Coghill D, Sonuga-Barke EJS (2012) Annual research review: categories versus dimensions in the classification and conceptualisation of child and adolescent mental disorders-implications of recent empirical study. J Child Psychol Psychiatry 53(5):469-489

4. Kraemer HC (2007) DSM categories and dimensions in clinical and research contexts. Int J Methods Psychiatr Res 16(Suppl 1):S8-S15

5. Regier DA (2007) Dimensional approaches to psychiatric classification: refining the research agenda for DSM-V: an introduction. Int J Methods Psychiatr Res 16(Suppl 1):S1-S5

6. Insel T, Cuthbert B, Garvey M, Heinssen R, Pine DS, Quinn K, Sanislow C, Wang P (2010) Research domain criteria (RDoC): toward a new classification framework for research on mental disorders. Am J Psychiatry 167(7):748-751

7. Robel L, Rousselot-Pailley B, Fortin C, Levy-Rueff M, Golse B, Falissard B (2013) Subthreshold traits of the broad autistic spectrum are distributed across different subgroups in parents, but not siblings, of probands with autism. Eur Child Adolesc Psychiatry. doi:10.1007/s00787-013-0451-5

8. Nilsson KW, Sonnby K, Nordquist K, Comasco E, Leppert J, Oreland L, Sjöberg RL (2013) Transcription Factor Activating Protein- $2 \beta$ (TFAP-2 $\beta$ ) genotype and symptoms of attention deficit hyperactivity disorder in relation to symptoms of depression in two independent samples. Eur Child Adolesc Psychiatry. doi:10.1007/s00787-013-0450-6

9. Fuhrmann P, Equit M, Schmidt K, von Gontard A (2013) Prevalence of depressive symptoms and associated developmental disorders in preschool children: a population-based study. Eur Child Adolesc Psychiatry. doi:10.1007/s00787-0130452-4

10. Theunissen SCPM, Rieffe C, Kouwenberg M, De Raeve LJI, Soede W, Briaire JJ, Frijns JHM (2013) Behavioral problems in school-aged hearing-impaired children: the influence of sociodemographic, linguistic, and medical factors. Eur Child Adolesc Psychiatry. doi:10.1007/s00787-013-0444-4

11. Mazzei DH, Martín Rodriguez S, Pérez Moltó H, Ruíz Izquierdo J, Baeza I (2013) A forgotten lethal psychosis: a case report. Eur Child Adolesc Psychiatry. doi:10.1007/s00787-0130449-z 
12. Posserud M-B, Ullebø AK, Plessen KJ, Stormark KM, Gillberg C, Lundervold AJ (2013) Influence of assessment instrument on ADHD diagnosis. Eur Child Adolesc Psychiatry. doi:10.1007/ s00787-013-0442-6

13. Hall CL, Moldavsky M, Taylor J, Sayal K, Marriott M, Batty MJ, Pass S, Hollis C (2013) Implementation of routine outcome measurement in child and adolescent mental health services in the
United Kingdom: a critical perspective. Eur Child Adolesc Psychiatry. doi:10.1007/s00787-013-0454-2

14. Hebebrand J, Antel J (2014) Squaring the circle? On the search for circulating biomarkers in polygenic psychiatric disorders. Eur Child Adolesc Psychiatry 23(3):123-125. doi:10.1007/s00787014-0527-x 